\title{
Performance Evaluation of Plain Weave and Honeycomb Weave Electrodes for Human ECG Monitoring
}

\author{
Xueliang Xiao, ${ }^{1}$ Sandeep Pirbhulal, ${ }^{2,3}$ Ke Dong, ${ }^{1}$ Wanqing $W u,{ }^{2}$ and Xi $\mathrm{Mei}^{2}$ \\ ${ }^{1}$ School of Textiles and Clothing, Jiangnan University, Wuxi 214122, China \\ ${ }^{2}$ Shenzhen Institutes of Advanced Technology, Chinese Academy of Sciences, Shenzhen 518055, China \\ ${ }^{3}$ Shenzhen College of Advanced Technology, University of Chinese Academy of Sciences, Shenzhen 518055, China
}

Correspondence should be addressed to Xueliang Xiao; xiao_xueliang@163.com and Wanqing Wu; 283077639@qq.com

Received 5 February 2017; Accepted 20 April 2017; Published 5 July 2017

Academic Editor: Young-Jin Cha

Copyright (c) 2017 Xueliang Xiao et al. This is an open access article distributed under the Creative Commons Attribution License, which permits unrestricted use, distribution, and reproduction in any medium, provided the original work is properly cited.

\begin{abstract}
Long-time monitoring of physiological parameters can scrutinize human health conditions so as to use electrocardiogram (ECG) for diagnosis of some human's chronic cardiovascular diseases. The continuous monitoring requires the wearable electrodes to be breathable, flexible, biocompatible, and skin-affinity friendly. Weave electrodes are innovative materials to supply these potential performances. In this paper, four conductive weave electrodes in plain and honeycomb weave patterns were developed to monitor human ECG signals. A wearable belt platform was developed to mount such electrodes for acquisition of ECG signals using a backend electronic circuit and a signal transfer unit. The performance of weave ECG electrodes was evaluated in terms of skin-electrode contacting impedance, comfortability, ECG electrical characteristics, and signal fidelity. Such performances were then compared with the values from $\mathrm{Ag} / \mathrm{AgCl}$ reference electrode. The test results showed that lower skin-electrode impedance, higher R-peak amplitude, and signal-to-noise ratio (SNR) value were obtained with the increased density of conductive filaments in weave and honeycomb weave electrode presented higher comfort but poorer signal quality of ECG. This study inspires an acceptable way of weave electrodes in long- and real-time of human biosignal monitoring.
\end{abstract}

\section{Introduction}

In the past decade, many kinds of wearable health monitoring systems have been developed in hospitals and reallife circumstances [1-3]. The wearable sensing hardware platform assists in acquiring and processing of multiple physiological features to provide an early recognition of pathological symptoms and to improve the healing rate of disease without superseding in patient's daily routine [4-6]. Electrocardiogram (ECG) has a noteworthy significance in all biosignals from human body. ECG contains consequential information about the individual heart and performance [7] and is a main investigated element for people with cardiovascular diseases and is a standard process in recent cardiac medical care $[8,9]$. To date, a large amount of efforts have been accomplished to formulate the approaches for easy, comfortable, accurate, flexible, and continuous monitoring of ECG signals from human body. The cardiovascular diseases are the main prevalent and severe life-threatening health issues in the world and the major foundation and reason of death for the people in the ages between 44 and $64[10,11]$.

To date, electrodes using $\mathrm{Ag} / \mathrm{AgCl}$ are mostly and widely utilized in medical environment for measuring ECG because of $\mathrm{Ag} / \mathrm{AgCl}$ 's low and stable half-cell potential. However, the external layer of human skin has a dry dielectric coat known as "stratum corneum," leading to a transporting diminution from ions to electrons. Therefore, $\mathrm{Ag} / \mathrm{AgCl}$ electrode cannot be exercised directly; a conductive gel is thus employed to moisturize the skin external layer in order to connect the cardiac microcurrent and the back-end monitoring circuit. Nevertheless, this gel-coating process is relative cumbersome and may bring difficulties in production. Moreover, there is likelihood that there may be gel residues on skin that may cause a short circuit of electrodes. Another drawback of using $\mathrm{Ag} / \mathrm{AgCl}$ electrode is its short serve time that cannot be used for long-time monitoring of human in 


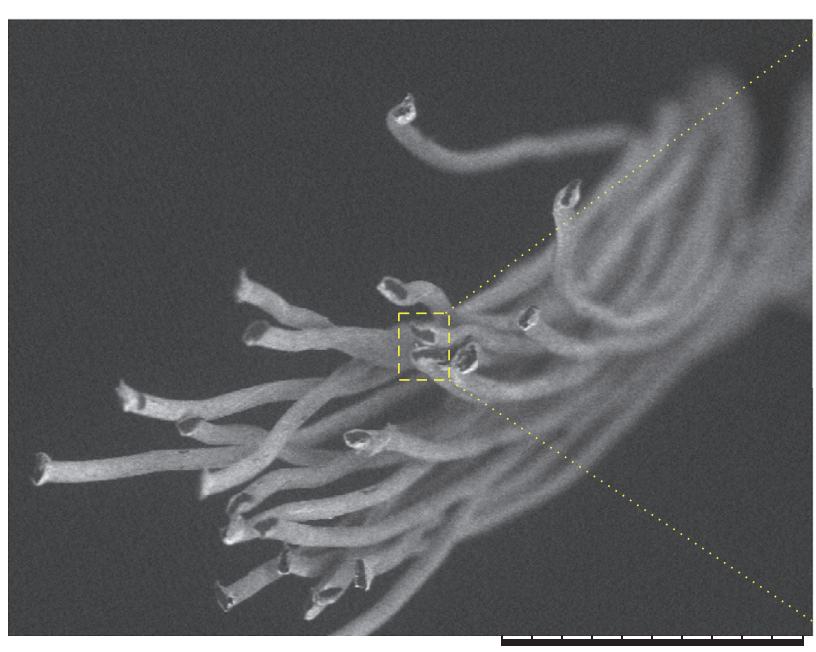

HL $\quad$ D5.4 $\times 250 \quad 300$ um

(a)

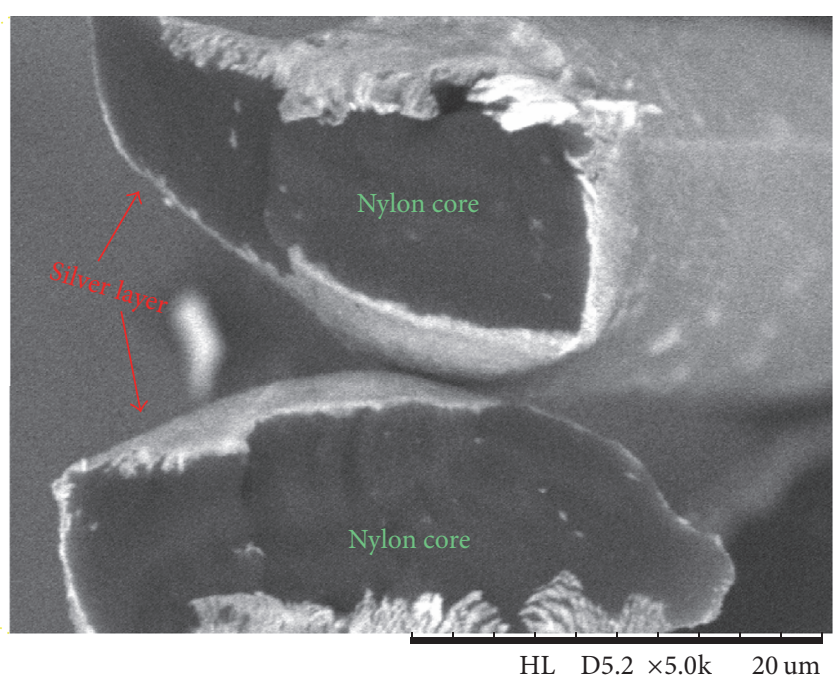

(b)

Figure 1: Conductive filaments, (a) a bundle of silver-coated nylon filaments used in woven fabric, and (b) an enlarged image of silver-coated layer on filament.

sleeping or working conditions $[12,13]$. In addition, $\mathrm{Ag} / \mathrm{AgCl}$ electrode for ECG measurement process is uncomfortable and painful for patients because the skin preparation for attaching such electrode habitually occupies an abrasion to the external skin layer. The patients who require frequently ECG measurements with skin preparations and gel utilization may also be a reason of infection or allergy. The signal quality of service (QoS) is usually degraded over duration because the skin stratum corneum cells reproduce and the coated conductive gel dries. Furthermore, another disadvantage of such electrodes is not reusable, and the recycling process requires plenty of time and common efforts [14-17].

However, these inherent weaknesses of $\mathrm{Ag} / \mathrm{AgCl}$ electrode may be overcome with the assistance of textile electrodes because textile structure can assemble different conductive materials within flexible and variable patterns [1820]. Because of the porous structure, textile electrode can offer better electric conductivity and comfortability without using gel coating. Cho et al. [21] introduced a sputtering conductive alloy on woven fabrics and compared the performance of different types of electrodes from embroidering and weaving processes. It was concluded that the performance of fabric with metal fiber composite $(\mathrm{Cu} / \mathrm{Ni}$ coated fiber) as a base and embroidered with the wire showed better results in electrical characteristics. A motion sensor was also integrated within the fabric in order to detect the change of skin-electrode impedance accurately. Kannaian et al. [13] proposed an approach of using embroidery stitch on the surface of polyester fabric with $75 \mathrm{D}$ longitudinal density and $150 \mathrm{D}$ horizontal density for electrodes using Wilcom Embroidery software. Such electrodes were compared with commercial $\mathrm{Ag} / \mathrm{AgCl}$ electrode for the performances in terms of electrode position, cleaning, signal quality, spin supplies, and repeatability. Zieba et al. [22] discussed a textronic knitting method that is integration of biosensors and measurement prototype within knitting patterns. The knitted fabric was used in their work, and the platform for ECG monitoring was based on three-electrode method. The performance of such functional fabric was compared with different electrodes in ECG monitoring. In the above studies, researchers have devoted to discover more types of conductive and comfortable ECG electrodes. However, the effects of electrode pattern and structure on the electrode conductivity, wearable comfort, and quality of ECG signals have not been studied yet.

This paper introduces four weave-based ECG electrodes with different contents of conductive yarns and weave patterns in ECG acquisition. The weave-based electrodes include plain weave and honeycomb weave with different flexibility, touch feeling, air permeability, and area electrical resistance, respectively. A wearable hardware platform for mounting weave electrodes is used for ECG monitoring. The skin-electrode interference impedance, signal-to-noise ratio (SNR), comfortability from different subjects, and other electrical characteristics of ECG are captured and compared finally, including a comparison between the developed weave electrodes and the reference $\mathrm{Ag} / \mathrm{AgCl}$ electrodes for the performance in ECG monitoring.

\section{Methods and Materials}

2.1. Preparation of Weave-Based Electrode. In comparison with knitted fabric, woven fabric is a more stable candidate in biosignal monitoring that is important for reducing motion artifact and obtaining stable signals. In weave patterns, plain weave and honeycomb weave show two typical styles for different comfort parameters, such as air permeability, touch feel, heat, and moisture transfer. Moreover, conductive filaments in the patterns have different bulk resistance evidently even under the same warp and weft densities. Here, we prepared the conductive woven fabrics using silver-coated nylon filaments (70 D, Danier, a unit for fineness). The coated silver layer on each filament has a thickness range of 100 $200 \mathrm{~nm}$, as shown in Figure 1. It was measured that the 


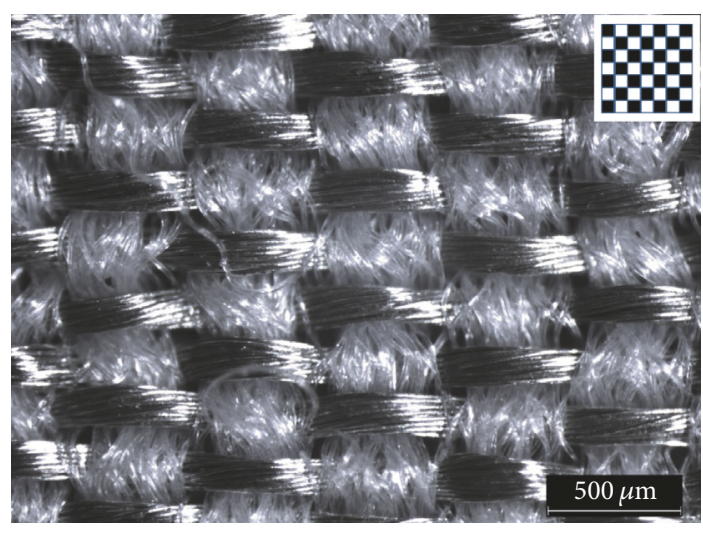

(a)

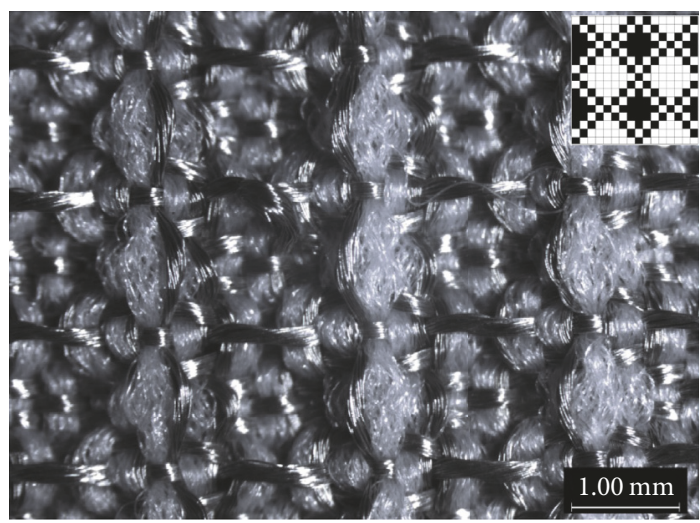

(c)

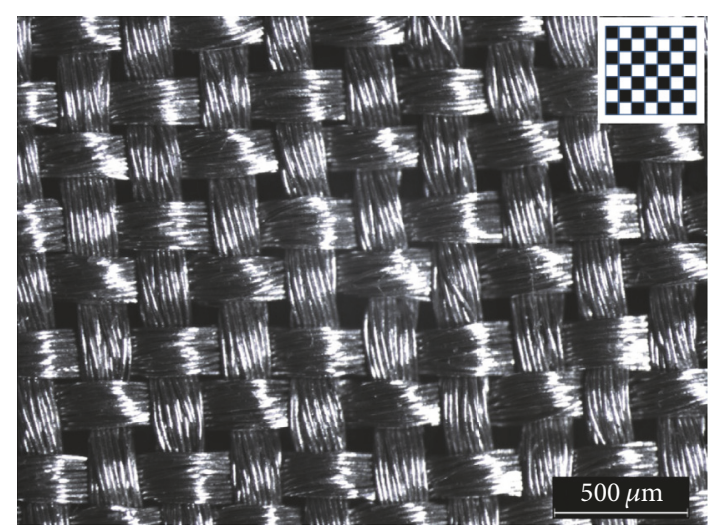

(b)

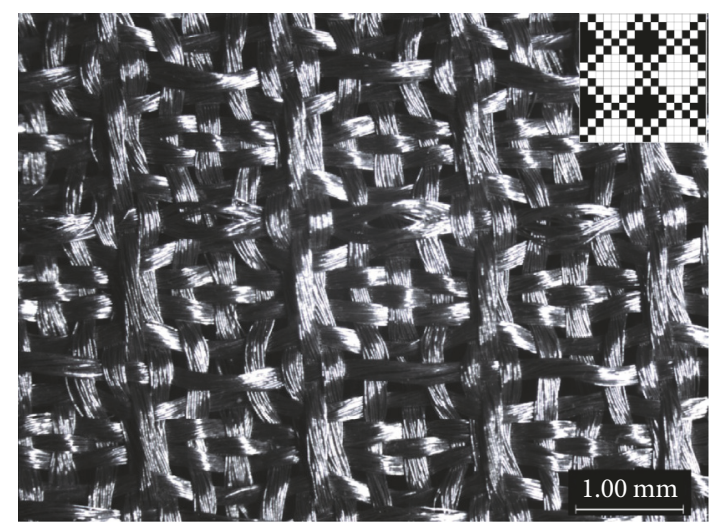

(d)

FIGURE 2: Description of four developed weave-based electrodes: (a) plain woven fabric E1 with conductive wefts and nonconductive warps, (b) plain woven fabric E2 with pure conductive yarns, (c) honeycomb woven fabric E3 with conductive wefts and nonconductive warps, and (d) honeycomb woven fabric E4 with pure conductive yarns. The right-top inset figure in each image shows the weave pattern of corresponding fabric.

TABLE 1: Specifications of four developed woven fabrics.

\begin{tabular}{lccc}
\hline Fabric & Warp density $($ yarns $/ \mathrm{cm})$ & Weft density $($ yarns/cm) & Thickness $(\mathrm{mm})$ \\
\hline Plain woven fabric $a$ & 20 & 64 & 0.45 \\
Plain woven fabric $b$ & 37.35 & 42.71 & 0.32 \\
Honeycomb fabric $a$ & 27.03 & 38.57 & 1.78 \\
Honeycomb fabric $b$ & 30 & 30 & 1.06 \\
\hline
\end{tabular}

electrical resistance of each bundle of filaments is $0.02 \sim$ $0.03 \Omega / \mathrm{cm}$. Another kind of yarn used in woven fabric is nonconductive, which is made of blended nylon filaments and cotton fibers $(70 / 30,70 \mathrm{D})$.

A rapier woven loom (SL8900S, automatic weave machine) is employed to manufacture four woven fabrics for textile electrodes with the mentioned patterns and materials. These weave electrodes were tailored into identical square size of $45 \times 45 \mathrm{~mm}^{2}$ to avoid interferences for signal collection, which is referred to the commercial disposable circular electrode (such as $3 \mathrm{M}$ electrode) with diameter of $45 \mathrm{~mm}$. Two plain woven fabrics have the same weft yarns that are bundles of silver-coated nylon filaments with twist of $1 / \mathrm{cm}$, as shown in Figures 2(a) and 2(b). The warp yarns of the two plain woven fabrics are different, for example, conductive and nonconductive, which endow the two fabrics with different area resistances. The honeycomb woven fabrics show similar situations with plain woven fabrics. The specifications of the four fabrics are listed in Table 1.

The fabric morphologies were characterized using a Leica M165C microscope; the fabric thickness was measured by a standard tester $\left(6 \mathrm{~g} /\right.$ inch $^{2}$ pressure). Figure 2 shows the SEM images of as-made woven electrodes. Plain woven fabrics show relatively flat surface with regular pores at the interlaced points of four neighbor warp and weft yarns. The size of pores or fabric porosity decides the fabric permeability and wearable comfortability. Honeycomb woven fabric shows slightly a periodicity and forming repeats of inverted pyramidal space on the both surfaces of fabric. In a honeycomb fabric as shown in Figure 2(c), the part of a warp yarn underneath 


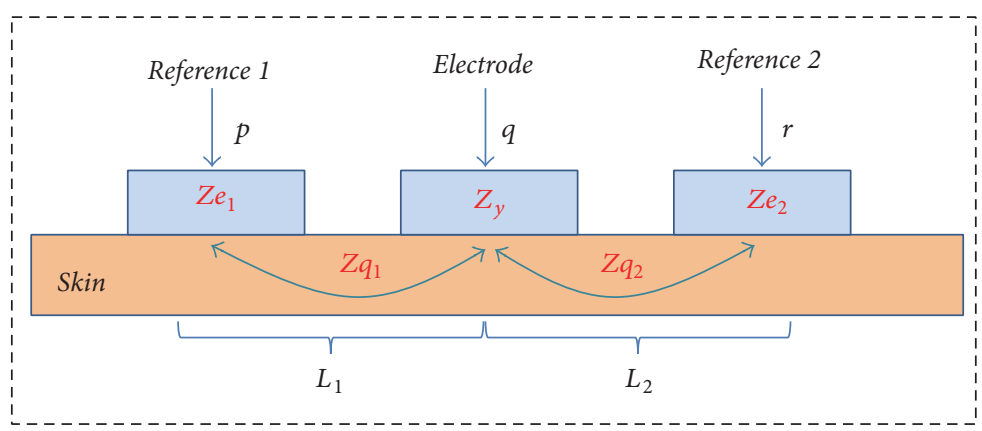

(a)

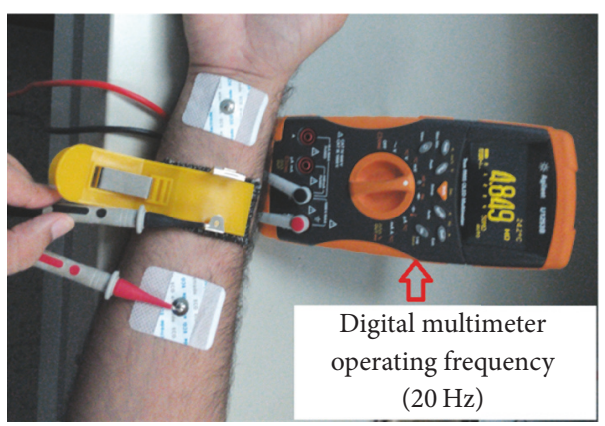

(b)

FIGURE 3: Measurement of skin-electrode impedance, (a) block diagram, and (b) real setup for measurement of electrochemical impedance.

a weft float is a warp float. Two layers of floats form a closed internal space. Owing to the periodical repeats of the inverted pyramidal space and the closed internal space, the fabric has been reported with good sound and moisture absorption, good insulating, and quick dry rate $[23,24]$. The as-made honeycomb woven fabrics show different electric resistance due to different contents of conductive filaments and connection of fabric surface to human skin.

2.2. Skin-Electrode Impedance Measurement. Impedance measurement is the most important step for evaluating the performance of weave-based electrodes in acquisition of biosignals from human body. To compare the conductivity of different weave-based electrodes, a digital multimeter was used to measure the skin-electrode impedance. A commercial $\mathrm{Ag} / \mathrm{AgCl}$ ECG electrode (made in 3M Group) was used as a reference electrode [10]. A skin-electrode model using equivalent circuit is shown in Figure 3. The skin-electrode impedance can be calculated as

$$
\begin{aligned}
& Z_{p q}=Z_{q 1}+Z_{e 1}+Z_{y}, \\
& Z_{q r}=Z_{q 2}+Z_{e 2}+Z_{y}, \\
& Z_{p r}=Z_{q 1}+Z_{q 2}+\left(Z_{e 1}+Z_{e 2}\right),
\end{aligned}
$$

where $Z_{q 1}$ and $Z_{q 2}$ denote the body impedance between a reference and an unknown electrode at the points " $p, q$ " and " $q, r$," respectively. $Z_{e 1}$ and $Z_{e 2}$ stand for the interface impedance between the skin and reference electrode at the points of $q$ and $r$ respectively. $Z_{y}$ is the interface impedance between skin and unknown electrode. $Z_{p q}, Z_{q r}$, and $Z_{p r}$ are the impedance between each electrode. The proposed weave electrode, in this case, is the unknown electrode. $Z_{y}$ is located at the center between the two reference electrodes; suppose that $L_{1}=L_{2}$ [7]. Since the same reference electrode was used, it is assumed that

$$
\begin{aligned}
& Z_{e 1}=Z_{e 2}, \\
& Z_{q 1}=Z_{q 2},
\end{aligned}
$$

$$
\begin{aligned}
& Z_{y}=Z_{p q}-\frac{Z_{p r}}{2}, \\
& Z_{y}=Z_{q r}-\frac{Z_{p r}}{2} .
\end{aligned}
$$

Under frequency of $20 \mathrm{~Hz}$, the digital multimeter was utilized to evaluate the impedance between the test points " $p$ " and " $q$," " $q$ " and " $r$," and " $p$ " and " $r$," respectively. The electrode impedance $Z_{y}$ was calculated using (3) or (4). During the measurement of impedance of a weave electrode, it should be pointed out that the impedance tends to decrease due to the increased contacting area of electrode and human skin that is caused by compression of conductive yarns to skin under a certain pressure. Herein, an elastic Velcro was utilized to offer a stable pressure up to $1000 \mathrm{~N} / \mathrm{m}^{2}$.

2.3. Establishment of Wearable System for ECG Signal Acquisition. In order to compare the ECG signals acquired through the developed weave electrodes fairly, a dedicated wearable system for recording biopotentials has been designed and established, as shown in Figures 4 and 5. The system consists of three components, namely, sensor (mainly electrodes), integrated hardware platform, and display terminal, as shown in block diagram of Figure 4. Among the three parts, the main body is the integrated wearable hardware platform that contains a HKD-10A module, signal filter, analogdigital conversion, and a wireless communication module. The whole platform is constructed through a filtering and amplification circuit. To reduce the obtained signal noises, such as the noises from baseline drift or $50 \mathrm{~Hz}$ of power interference, a developed recording unit is designed specifically and manufactured for ECG monitoring using a HKD-10A monolithic analog front-end with high gain and 1-T $\Omega$ input impedance. Owing to the limitation of power consumption, a MSP430F149 module is adopted as a data processor. A HKD-10A sensor was designed to collect ECG signals from microcontroller when an enabling signal was sent from a chip microcontroller through a UART port. The port can be used to stream data into a PC through a Bluetooth communication protocol or an oscilloscope through a connecting probe. The energy for operating the whole system is supplied by a rechargeable Li-ion battery $(3.3 \mathrm{~V})$ or a rechargeable USB terminal [25]. 


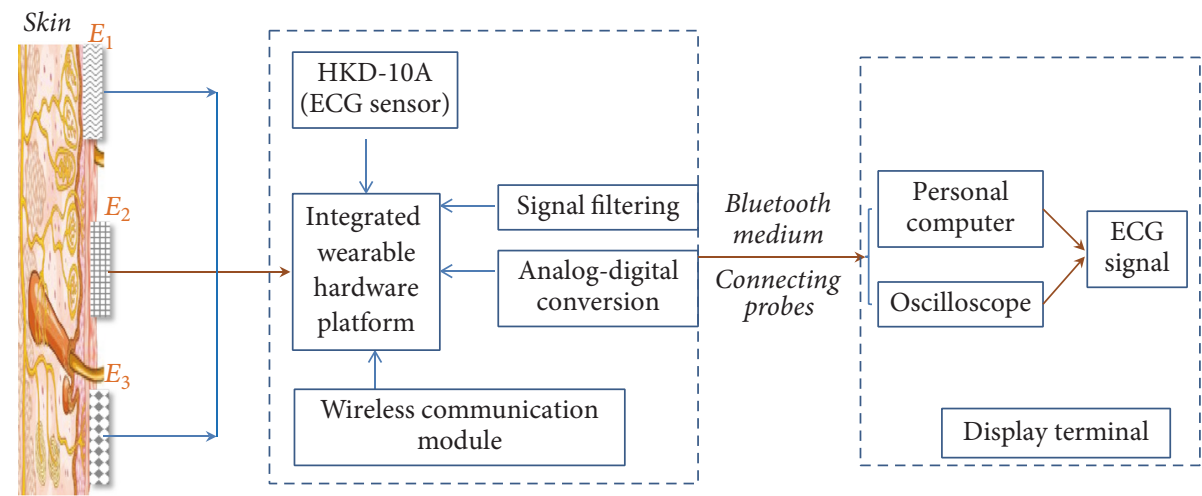

FIGURE 4: A block diagram for wearable hardware platform implementation for obtaining ECG from skin to display terminal.

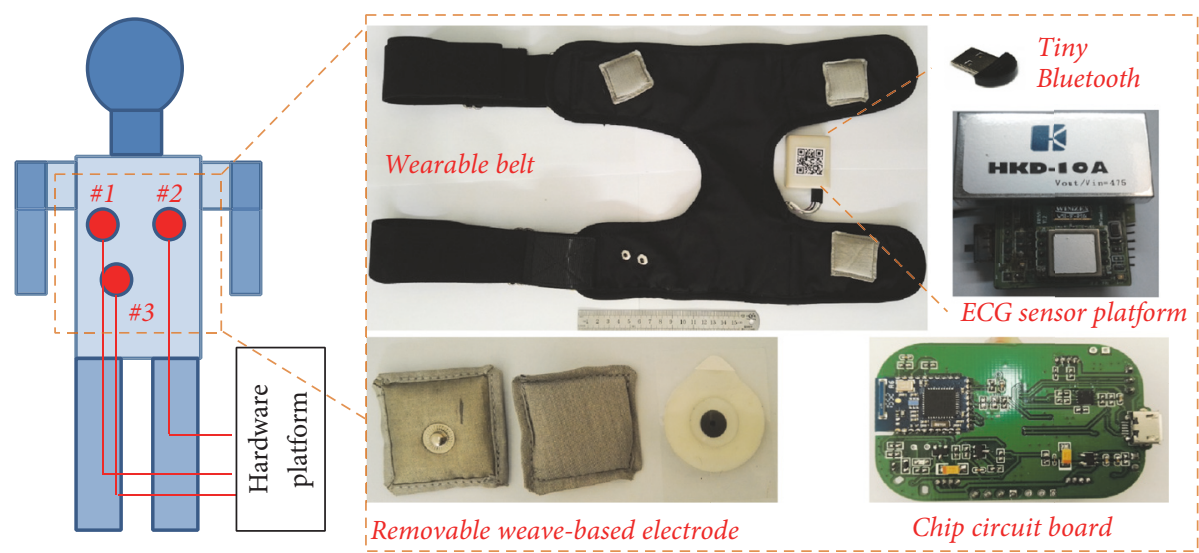

FIGURE 5: Three points for human-hardware platform interaction for ECG measurement and the real wearable experiment setup using belt format and components mounted on the hardware platform.

Noise is the biggest trouble in obtaining clear and stable ECG signals. The noise is generated mainly due to the power line interference, electrode contact noise, baseline drift, instrumentation noise, motion artifacts, electrosurgical noise, and other less significant noise sources. Generally, a hardware filter can eliminate unwanted noise. The filter restricts those noise frequencies where noise occurred, remaining the useful frequencies of ECG signals. The major restriction of hardware filter depends on capacitors that cannot be proscribed well both in construction and in normal utilization [26-29].

Apart from the hardware platform, we also developed software that helps to monitor ECG. Owing to the scrawny shape of ECG signals that can be easily corrupted by various kinds of noises that cannot be filtered completely by the hardware filter, a software filter is dependent on the cutoff frequencies that can be controlled precisely by allowing implementation of advanced filter models. Particularly, ECG amplitude is extremely small, approximately at $1 \mathrm{mV}$. Thus, it is essential to exercise filter to eliminate an extensive range of noises. Therefore, at the software end, a digital notch filter at $60-\mathrm{Hz}$ for minimizing the power line interference, a finite impulse response band-pass filter for correcting baseline wander, a multiscale mathematical morphology filter for eliminating motion artifacts and power line interference, and a differential operation method for smoothing and normalizing have been integrated into this developed platform. The ECG signals were recorded under a speed of $25 \mathrm{~mm} / \mathrm{s}$. Moreover, the wearable platform is in the format of wearable belt, as shown in Figure 5, where four points are available to mount the removable weave-based electrodes. The developed conductive woven fabrics are tailored into pillow-block shape $\left(4 \times 4 \mathrm{~cm}^{2}\right)$ of electrodes that filled with a very thin layer of sponge. The removable electrodes connect with the hardware platform through metal buttons.

2.4. Weave-Based Electrode for ECG Monitoring. The ECG monitoring of a human was carried out using a standard Holter with a single lead, as shown in the three-point interaction of diagram in Figure 5. The obtained lead is approximately oriented along the cardiac axis. This method is a way to produce high quality of ECG because the typical orientation of the cardiac axis is well-known in clinical option. Additionally, this method of standard placement has an intrinsic advantage in conveying useful information for inspection. In this study, $\mathrm{Ag} / \mathrm{AgCl}$ electrode was used as a 
TABLE 2: Fundamental base for performance evaluation of developed weave electrodes for ECG monitoring.

\begin{tabular}{lccc}
\hline Particulars & & Basis of evaluation & \\
QRS-complex & 2 points & 1 point & points \\
\hline P-wave & Found & - & Not found \\
T-wave & Found & - & $<0.3-0.35 \mathrm{~V}$ \\
R wave amp & $>0.35 \mathrm{~V}$ & $10 \mathrm{~s}-60 \mathrm{~s}$ & $>0.3 \mathrm{~V}$ \\
Stabilization time & $\leqslant 10 \mathrm{~s}$ & $0.05-0.1 \mathrm{~V}$ & $>60 \mathrm{~s}$ \\
R-peak amplitude variation & $<0.05 \mathrm{~V}$ & & $>0.1 \mathrm{~V}$ \\
\hline
\end{tabular}

TABLE 3: Weighting coefficients for result analysis.

\begin{tabular}{lcccccc}
\hline Particulars & QRS-complex & P-wave & T-wave & R wave amp & Stabilization time & R-peak amp variation \\
\hline Weight coefficient & $20 \%$ & $20 \%$ & $20 \%$ & $20 \%$ & $10 \%$ & $10 \%$ \\
\hline
\end{tabular}

ground reference for measuring impedance. Weave-based electrodes and standard Ag/AgCl electrodes were placed side by side for simultaneous recording the biosignals.

Here in detail, two electrodes were positioned on the chest surface (\#1 and \#2) near to heart, while a third reference electrode (\#3) was placed on the lower left position that is $10 \mathrm{~cm}$ away from electrode \#1. This arrangement enables producing relatively neat and clean ECG signals with higher R-peak amplitude and QRS-complex waves. For a specific weave-based electrode, its one fabric side was attached to human skin, while the other side was connected to the developed wearable belt through the metal buttons. The acquired signals can be visualized and stored to a specific base station.

2.5. Evaluation of Electrode's Comfortability. The comfort level is a very important factor for patient to accept the attached electrodes for long-time biosignal monitoring. Different types of electrodes have distinguished levels of comfort, because it varies from structures, densities, and materials. There are several subjective comfort performance indexes, for example, thermal comfort, tactile comfort, style comfort, and pressure comfort. To monitor the human ECG, the tactile (touch feeling) comfort is a very useful parameter because the wearable platform requires long time to monitor. The tactile comfort depends on the microstructure, fabric density, and fiber properties of weave-based electrodes. The tactile comfort can be measured on the basis of comfortable feeling to skin-electrode contact. The discomfort is mainly due to tickle, itch, prickle, scratchiness, fiber shedding, and all other factors that could irritate the patients during the long-time ECG monitoring. This study involves four developed weavebased electrodes; each one has a unique comfortability. For testing the comfort level of each electrode, the electrode was attached on the wrist of participant for friction experiment. After the process, each participant was asked about the feeling and comfort level; this subjective feeling was recorded with average value by the informed written consent that was provided to all participants before undergoing the ECG monitoring using the developed electrodes. The other objective parameters of comfort levels, such as thermal conductivity and air permeability, to some extent can tell us the comfortability of developed weave-based electrodes. Here, air resistance and thermal conductivity were measured five times for average values. The instruments are KES-F Thermo Labo II for measuring the thermal conductivity and KES-F8-AP1 air permeability tester for measuring the air resistance of electrodes.

2.6. Process of Human ECG Monitoring. Ten right-handed volunteers without history of cardiovascular diseases were employed for our ECG monitoring experiments. Each subject was tested under the standard conditions between 4:00 pm and 10:00 pm at temperatures of $21-26^{\circ} \mathrm{C}$ after refraining from smoking and coffee utilization for six hours before his/her contribution to the experiment. The investigated properties of ECGs and basis of evaluation can be viewed in Table 2 . Many factors influence the evaluation of electrode functional quality. Therefore, an evaluation method was developed to compare different weave-based electrodes with different weighting coefficients.

In a repeat of ECG, QRS-complex, P-wave, and Twave are the main parts that can describe the action of heart. In the evaluation of electrode's quality, if these wave parts were clearly visible, then 2 points were given for the electrode quality; otherwise 0 points were given for the electrode quality without clear waves. The stabilization time was measured from $0 \mathrm{sec}$ until the baseline settled to the level of $1.6 \mathrm{~V}$. If the stabilization time was shorter than 10 seconds, 2 points were then given for the evaluation of electrode quality; however, if the stabilization time was in the range of 11 to 60 seconds, 1 point was given for related electrode quality; otherwise, 0 point was given for related electrode quality. The R-peak amplitude means the signal strength. If it is over $0.35 \mathrm{~V}$, then it will obtain 2 points; if it is under $0.01 \mathrm{~V}$, then it will obtain 0 points. The R-spike amplitude variation means the difference $(\mathrm{V})$ between the lowest and highest peaks. If amplitude variation is under $0.05 \mathrm{~V}$, then it gives 2 points and if it is over $0.1 \mathrm{~V}$, then it will obtain 0 points. The basis of evaluation standard is listed in Table 2.

As shown in Table 3, weighting coefficients were used that depends on the importance of the electrode property in the long-time ECG monitoring and analysis. Here, the most important properties (weighting coefficient) in the 


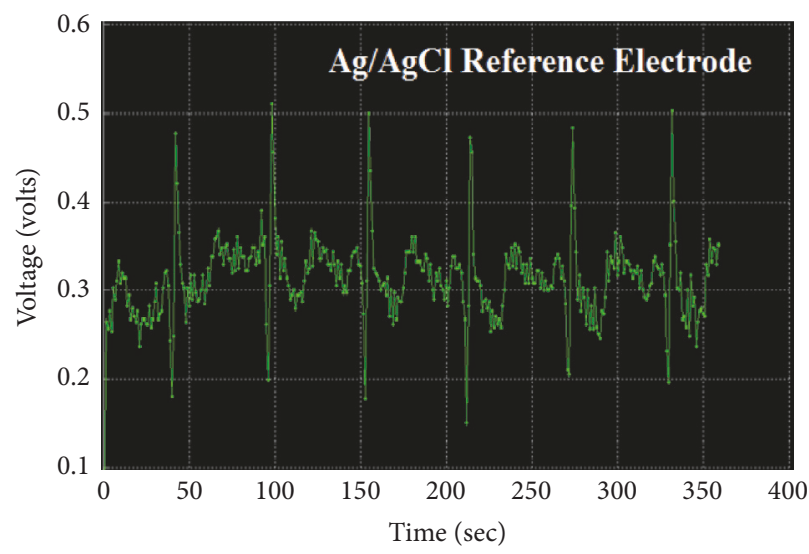

(a)

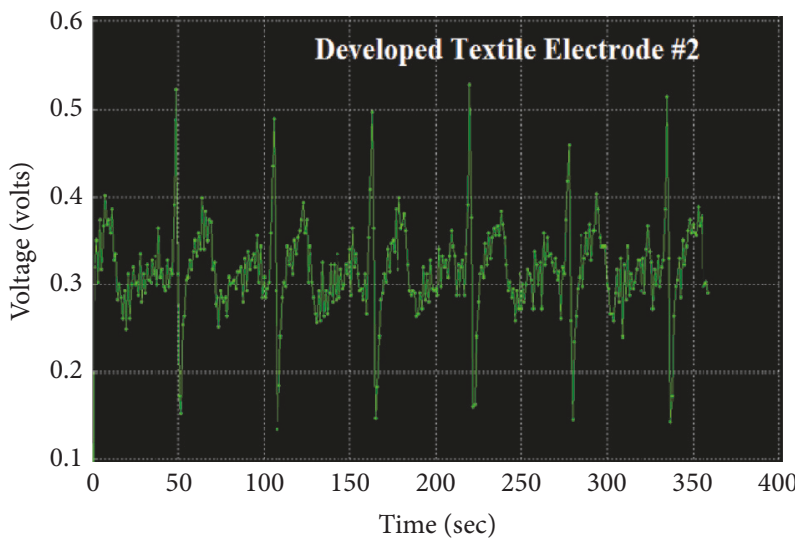

(c)

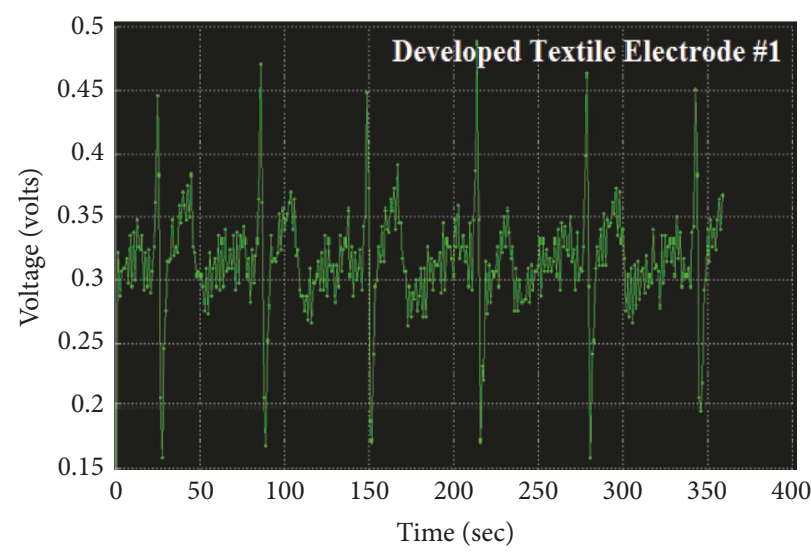

(b)

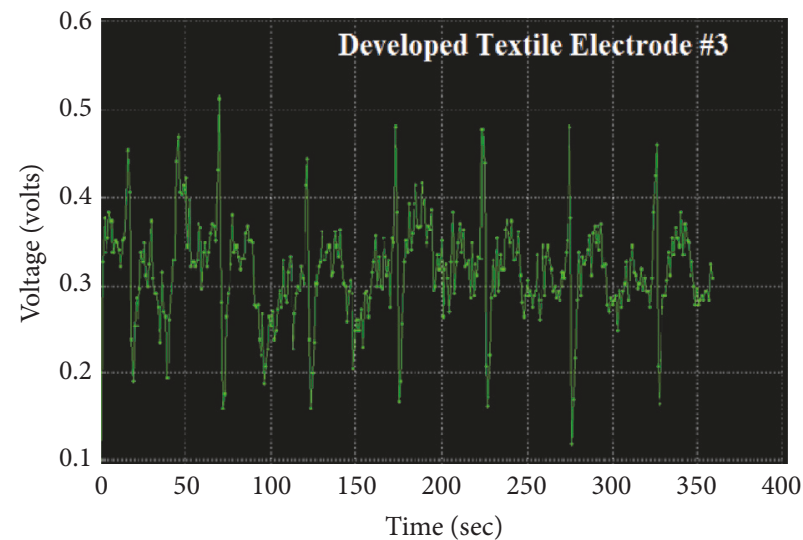

(d)

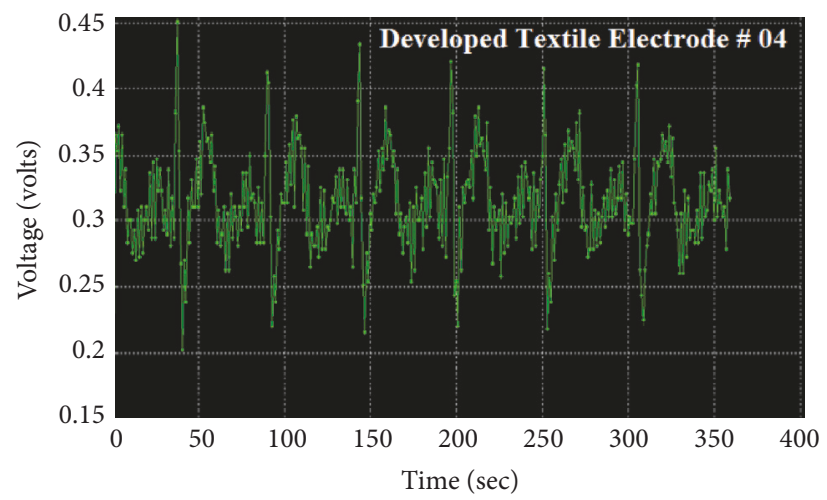

(e)

FIGURE 6: Real-time ECG signals obtained from different electrodes: (a) Ag/AgCl reference electrode; (b) plain woven fabric E1; (c) plain woven fabric E2; (d) honeycomb woven fabric E3; (e) honeycomb woven fabric E4.

evaluation are QRS-complex, P-wave, T-wave, noise, and $50 \mathrm{~Hz}$ interference. These properties are important because they affect the diagnosis of cardiovascular diseases mostly through ECG signals.

\section{Results and Discussion}

3.1. ECG Signals from Weave-Based Electrodes. Figure 6 shows five screenshots of ECG signal monitoring for the same person using five different electrodes that mounted on the developed ECG system. Compared with the quality of ECG signals, it can be judged easily that honeycomb woven fabrics give poorer ECG signals than the signals from plain weaves because some large voltage ranges of $\mathrm{P}$ and $\mathrm{T}$ waves appeared through honeycomb electrodes. This may be due to the different skin-electrode interface impedance of electrode to skin. For plain weave electrode, fabric made of pure conductive filaments shows better signal quality in 


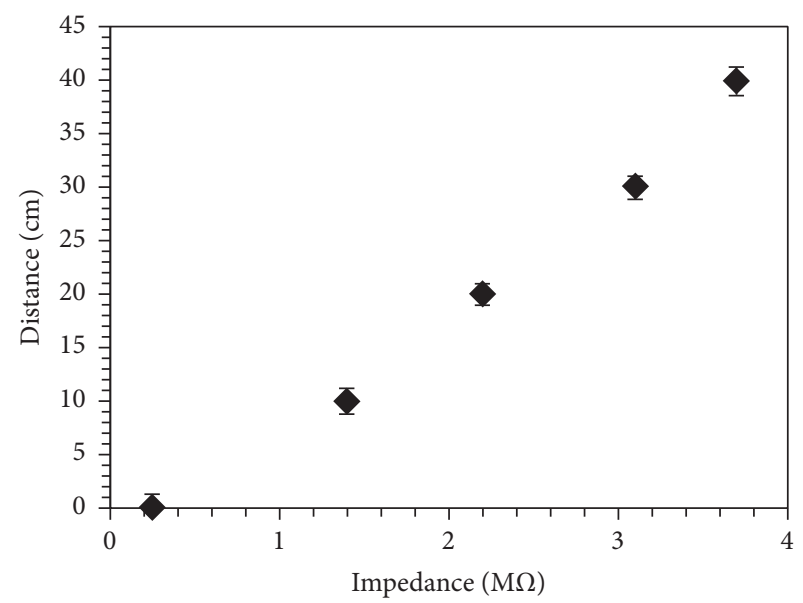

(a)

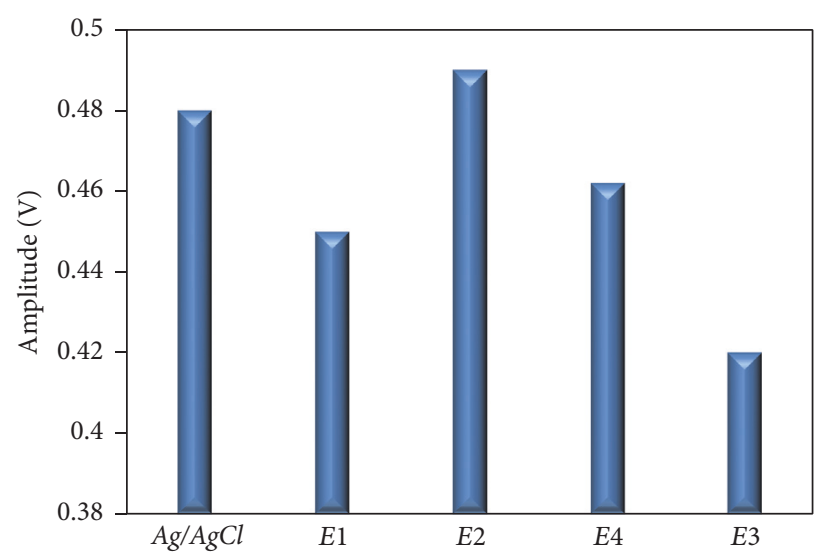

(c)

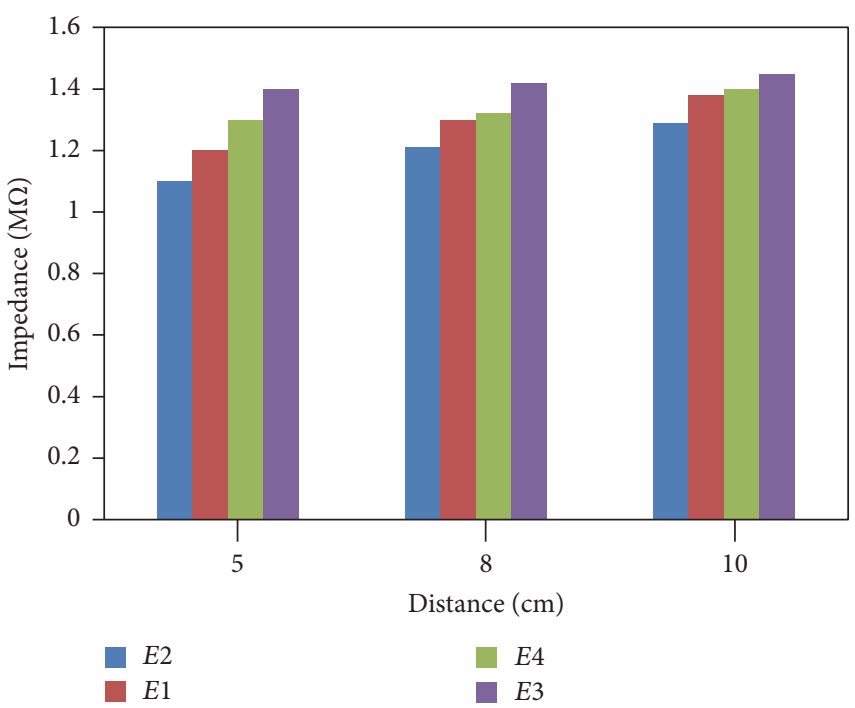

(b)

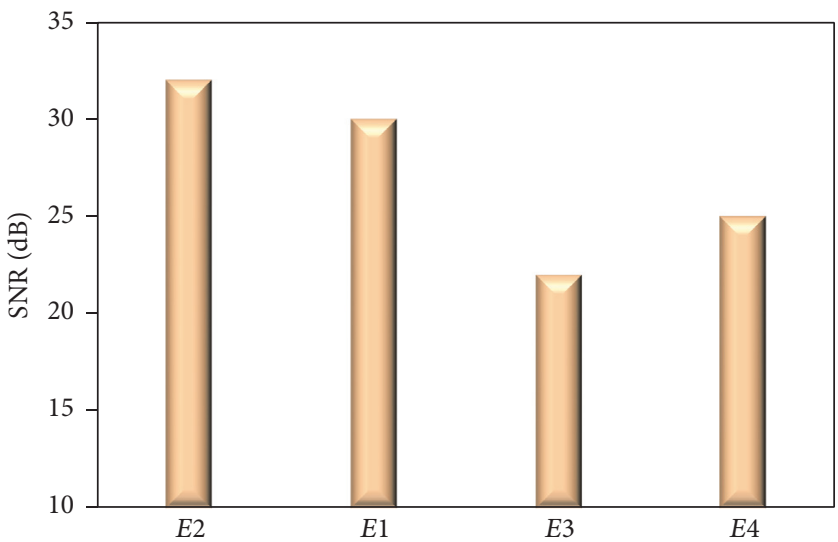

(d)

FIGURE 7: Experimental results: (a) effect of distance between two reference electrodes for measuring skin-electrode interference impedance, (b) skin-electrode impedance comparisons between developed electrodes, (c) R-peak amplitude comparisons between weave-based electrodes, (d) signal-to-noise ratio (SNR) comparisons between different weave-based electrodes.

ECG acquisition than the fabric interwoven by conductive weft and nonconductive warp filaments. This can be viewed from the amounts of noises and clarity of P-wave and T-wave in the real ECG signals. However, in comparison with the signal quality from $\mathrm{Ag} / \mathrm{AgCl}$ electrode, especially to compare the P-wave characteristic peak between Figures 6(a) and $6(\mathrm{c})$, plain weave electrode still shows slightly lower level in signal collection; even the collected ECG does not affect the diagnosis of patient's cardiovascular diseases.

Based on Figure 6, the acceptance of developed weavebased electrodes was compared according to six parameters and listed in Table 4 . From the final scores, it is noted that only electrode E2 has the same value with the reference $\mathrm{Ag} / \mathrm{AgCl}$ electrode, indicating that $\mathrm{E} 2$ can replace the commercialized electrodes from the viewpoint of ECG monitoring. Other developed weave-based electrodes, more or less, have some weakness in monitoring biosignals. The following sections will discuss about the detailed information of developed electrodes in specific performances.
3.2. Difference of Skin-Electrode Impedance among Electrodes. Obviously, textile electrodes can be used for long-term monitoring of biosignals because the electrodes do not annoy human skin; additionally, they are washable, lightweight, and ductile. However, a key disadvantage of such electrode is its large electrode-skin interference impedance. Normally, textile electrodes have impedance values with human skin in the range of $1-5 \mathrm{M} \Omega / \mathrm{cm}^{2}$ while the $\mathrm{Ag} / \mathrm{AgCl}$ electrode shows much less impedance up to $10 \mathrm{k} \Omega / \mathrm{cm}^{2}$. As shown in the experiment setup in Figure 3, the distance between each weave-based electrode and reference electrode can be changeable. The relationship between the distance and the measured related impedance can be plotted in a linear increase tendency, as shown in the tested data in Figure 7(a). This indicates that the human skin is an isotropic resistance when assuming the interface resistance of skin-electrode constant. A comparison for skin-electrode impedance is gathered up between the developed weave electrodes as shown in Figure 7(b). An increase relationship of the impedance 


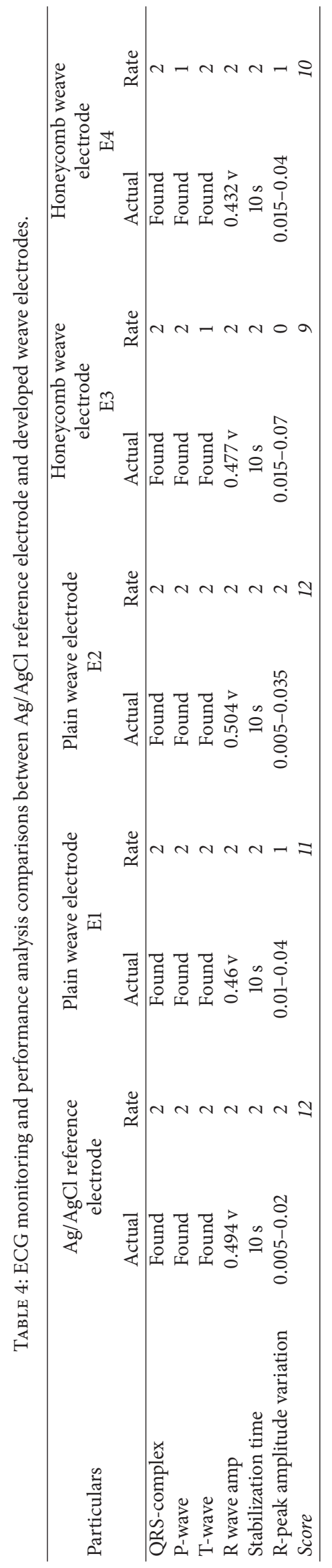


TABLE 5: R-peak amplitude comparisons between $\mathrm{Ag} / \mathrm{AgCl}$ reference and developed weave-based electrodes.

\begin{tabular}{|c|c|c|c|c|c|}
\hline \multirow[b]{2}{*}{ Number } & \multicolumn{5}{|c|}{ R-peak amplitude (V) } \\
\hline & $\begin{array}{l}\mathrm{Ag} / \mathrm{AgCl} \\
\text { reference } \\
\text { commercial } \\
\text { electrode }\end{array}$ & $\begin{array}{l}\text { Plain weave } \\
\text { electrode } \\
\text { E1 }\end{array}$ & $\begin{array}{c}\text { Plain weave } \\
\text { electrode } \\
\text { E2 }\end{array}$ & $\begin{array}{c}\text { Honeycomb } \\
\text { weave electrode } \\
\text { E4 }\end{array}$ & $\begin{array}{c}\text { Honeycomb } \\
\text { weave electrode } \\
\text { E3 }\end{array}$ \\
\hline 1 & 0.485 & 0.450 & 0.520 & 0.510 & 0.460 \\
\hline 2 & 0.510 & 0.470 & 0.490 & 0.440 & 0.420 \\
\hline 3 & 0.500 & 0.450 & 0.495 & 0.480 & 0.445 \\
\hline 4 & 0.480 & 0.485 & 0.520 & 0.480 & 0.430 \\
\hline 5 & 0.485 & 0.460 & 0.480 & 0.485 & 0.420 \\
\hline 6 & 0.505 & 0.450 & 0.520 & 0.470 & 0.420 \\
\hline
\end{tabular}

with the distance for each kind of weave-based electrode is still viewed. Under one distance, it is clearly found that plain weave electrode shows less impedance than honeycomb electrode under the same size of electrode. This is due to the honeycomb feature with large amounts of repeats of microconcave pits on the fabric surface that cannot contact human skin completely.

Under the same fabric pattern, the application of nonconductive yarns into fabric would increase the impedance significantly; for example, E2 (with nonconductive filaments) has very low resistance in weft direction while it is almost insulated in warp direction. In experiment, the impedance has been measured under different distance values, for example, $5 \mathrm{~cm}, 8 \mathrm{~cm}$, and $10 \mathrm{~cm}$. Consistent with Figure 7(b), the test results in detail showed that plain woven fabric (E2) made of pure conductive filaments, fabric (E1) made of blended conductive filaments, honeycomb fabrics (E4) made of pure conductive filaments, and honeycomb fabric (E3) made of blended conductive filaments have skin-electrode impedance of 1.107, 1.227, and $1.269 \mathrm{M} \Omega \mathrm{s}, 1.195,1.299$, and $1.349 \mathrm{M} \Omega \mathrm{s}, 1.315,1.325$, and $1.419 \mathrm{M} \Omega \mathrm{s}$, and $1.407,1.440$, and $1.454 \mathrm{M} \Omega \mathrm{s}$ at distance of $5 \mathrm{~cm}, 8 \mathrm{~cm}$, and $10 \mathrm{~cm}$, respectively. The values indicate that the introduction of nonconductive filaments into electrode may increase the wearable electrode comfortability; however, it may not be conducive to reduce the impedance in biosignal monitoring.

3.3. ECG Electrical Characteristic Comparisons. The electrical performance of weave-based electrode in ECG monitoring is evaluated and recorded, as the R-peak amplitude values tested for six times shown in Table 5 . The average values of the amplitude are compared in the histogram of Figure 7(c). The R-peak amplitude values were obtained under the same test conditions including the same hardware and subject. Thus, it is noted that plain weave electrode E2 shows the highest amplitude, indicating that pure conductive fabric with tight attachment to human skin can effectively decrease the skin-electrode impedance and improve the whole circuit in signal peak amplitude, which means that such electrode can acquire even weaker biosignals or more sensitive to weak surface potential accumulated from heart or muscle. Due to the concave structure of each unit cell of honeycomb woven fabric, the fabric attaching to human skin is not as tight as plain woven fabric, which means that the effective contacting area of skin and electrode is reduced and the R-peak amplitude is manifested to decrease either. Similarly, introduction of common textile fibers into conductive fabric, although this would increase the fabric wearable comfortability, can decrease the sensitivity of fabric electrode in ECG monitoring; here, the observed peak amplitude is thereafter decreased. This is consistent with the test data and comparisons of developed weave-based electrodes in skinelectrode impedance.

Moreover, the stabilization time for ECG signals is set to be $10 \mathrm{~s}$ for all compared electrodes. In Table 4, a variable, namely, R-peak amplitude variation is used to estimate the biosignal stability in all cases. Consistently, a variation in R-peak amplitudes for each electrode is noted that the electrodes E1, E2, E3, and E4 show that R-peak variation is in $0.01-0.04,0.005-0.035,0.015-0.04$, and 0.015-0.07, respectively. This means the monitored signals are smoother to present in ECG when the resistance of electrode is less, and higher conductive electrode would lead to higher quality of ECG signals.

3.4. Comparison for Signal-to-Noise Ratio (SNR). SNR of ECG is determined by the root mean square (RMS) of the isoelectric region. For ECG, the SNR is the ratio of useful signal power $\left(P_{\text {signal }}\right)$ to noise power $\left(P_{\text {noise }}\right)$, as expressed in

$$
\mathrm{SNR}=\log \frac{P_{\text {signal }}}{P_{\text {noise }}}=\log \frac{\left(A_{\text {signal }}\right)^{2}}{\left(A_{\text {noise }}\right)^{2}},
$$

where "SNR" denotes the signal-to-noise ratio and " $A$ " represents the mean square root of amplitude. Because of using different filters, the signal of power frequency with the range of $49.5 \mathrm{~Hz}$ to $50.5 \mathrm{~Hz}$ was removed. Thus, the SNR of ECG signal for each electrode was determined. The comparison of SNR values between developed weave-based electrodes is illustrated in Figure 7(d) and Table 6. The measured SNR values for electrodes E3 and E4 are lower than the values from electrodes E1 and E2, among which E2 shows the highest $\mathrm{SNR}$ value, that is, $33.86 \mathrm{~dB}$. This means that the plain weave with conductive warp and weft yarns has better signal clarity or less noise under the same monitoring environment and conditions. This is consistent with the monitored signals 
TABLE 6: Signal-to-noise ratio (SNR) comparison between developed weave-based electrodes.

\begin{tabular}{lccccc}
\hline Electrode type & Measurement & Value 1 & Value 2 & Average value & SNR (dB) \\
\hline Plain W-electrode E2 & Signal & 0.203 & 0.389 & 0.296 & 0.006 \\
& Noise & 0.003 & 0.009 & 0.301 & 33.86 \\
Plain W-electrode E1 & Signal & 0.189 & 0.413 & 0.010 & 0.065 \\
& Noise & 0.007 & 0.320 & 0.284 & 0.015 \\
Honeycomb W-electrode E3 & Signal & 0.268 & 0.017 & 0.286 & 25.54 \\
& Noise & 0.013 & 0.253 & 0.011 & 28.29 \\
Honeycomb W-electrode E4 & Signal & 0.320 & 0.009 & & \\
\hline
\end{tabular}

TABLE 7: Comparison of comfort level between four weave electrodes.

\begin{tabular}{lccc}
\hline Electrode number & $\begin{array}{c}\text { Tactile comfort } \\
(? / 10)\end{array}$ & $\begin{array}{c}\text { Air resistance } \\
(\mathrm{KPa} \cdot \mathrm{s} / \mathrm{m})\end{array}$ & $\begin{array}{c}\text { Thermal conductivity } \\
(\mathrm{W} / \mathrm{m} \cdot \mathrm{K})\end{array}$ \\
\hline E1 & $7.5 / 10$ & 0.82 & 0.058 \\
E2 & $7 / 10$ & 0.68 & 0.065 \\
E3 & $8.5 / 10$ & 0.18 & 0.018 \\
E4 & $8 / 10$ & 0.12 & 0.024 \\
\hline
\end{tabular}

from ECG in Figure 6. Assuming the hardware, software system and test conditions are constant during all ECG monitoring; high quality of ECG means good connection of skin and electrode. More connection area of skin and electrodes would lead to high SNR value because more biopotential/charges are collected through the connection area. For such case, electrode E2 has more effective and larger amounts of conductive filaments in touching human skin. In comparison, honeycomb woven fabrics display more noises during ECG monitoring because of less effective conductive filaments attaching to skin and easier deformation of such fabric structure that causes the effective connection area may be always changeable slightly. This is manifested through the frequently appeared noises at the $\mathrm{P}$-wave and $\mathrm{T}$-wave positions.

3.5. Comparison of Weave Electrodes on Comfort Level. The comfort level of developed weave-based electrodes attached on human skin is a very important parameter, which decides the acceptance of patients to the developed weave electrodes. This parameter also determines whether the developed electrode is comfortable enough to be used for long-term physiological signal monitoring. As to our developed four weave ECG electrodes, they were woven with different patterns, material components, fabric density, microstructure, and fiber properties; thus the four weave electrodes have different tactile comfort levels. In this study, ten volunteers were employed to evaluate the tactile comfort of attached electrodes. Their scores are listed in Table 7, which showed that honeycomb woven fabric has better tactile comfort, especially when honeycomb uses common textile fibers that have already proved to be biocompatible to human skin.

Air resistance means the ease of air and moisture to transfer through the fabric electrode. A higher value of such factor indicates a more difficult of air and moisture to transfer. The tested values show that honeycomb woven fabric has better ability of air and moisture to transfer in comparison with plain weave electrodes. The thermal conductivity shows the heat transfer ability of fabric; this factor is useful when the wearer is in the extreme conditions. The tested values of such factors indicate that the warmth retention of honeycomb weave is better than plain weave because the concave surface of honeycomb could store still air inside for less heat convection. In a word, honeycomb weave electrode seems a better candidate for ECG signal monitoring because of its comfortable wear from subjective and objective views. However, from the viewpoints of skinelectrode impedance, electrical characteristic, and SNR value, conductive plain weave indicates a better candidate for ECG signal monitoring. This discovery may inspire new design of textile electrode for human biosignal long-term monitoring, so as to combine the structures of plain woven and honeycomb woven characteristics.

\section{Conclusions}

Textile electrode becomes popular in current booming developed wearable electronics for its flexible, breathable, biocompatible, and conductive properties that can be used for long-time monitoring of some human chronic cardiovascular diseases. Weave electrode has relative stable structure that is usually encountered in some new wearable electronics. Here, four woven conductive fabrics were developed in two patterns and pure/blended conductive components to study their feasibility in real-time ECG monitoring.

Apart from preparation of weave-based electrodes, we also established a hardware-based platform for acquiring signals where all system and test conditions were assumed constant during the whole monitoring process. Skin-electrode impedance, electrical characteristic, signal-to-noise ratio, and comfort level are key factors to evaluate the feasibility of developed weave-based electrode in biosignals collection. 
Experimental data indicated that more content of conductive filaments and larger connection area of conductive fabric part to human skin would lead to lower skin-electrode impedance, larger R-peak amplitude, larger value of signal-to-noise ratio, but poorer comfortability. There is a necessity to find out a balance point between higher quality of ECG acquisition and wearable comfortability. There may be an intermediate pattern between plain weave and honeycomb weave that both can obtain good ECG signals and also can give the wearer good feeling of skin attaching in dressing. In near future, we are looking forward to these developed weave electrodes in long-time biosignal monitoring for chronic diseases. A longterm training is also required for patients with diseases such as obstructive sleep apnea, hypertension, negative emotion management, and stress in order management for their proper monitoring.

\section{Disclosure}

Xueliang Xiao and Sandeep Pirbhulal are co-first authors.

\section{Conflicts of Interest}

The authors declare no competing financial interests.

\section{Authors' Contributions}

The authors Sandeep Pirbhulal and Xueliang Xiao contributed equally.

\section{Acknowledgments}

This work was financially supported by the National Undergraduate Innovation Training Program (Grant no. 201610295020), Natural Science Foundation of Jiangsu Province (Grant no. BK20160157), Research Fund for Industry-University Cooperation of Jiangsu Province (Grant no. BY2016022-07), and Guangzhou Science and Technology Planning Project (Grant no. 2016A030310129).

\section{References}

[1] A. Rangarajan, "Emerging trends in healthcare adoption of wireless body area networks," Biomedical Instrumentation and Technology, vol. 50, no. 4, pp. 264-276, 2016.

[2] S. Pirbhulal, H. Zhang, S. C. Mukhopadhyay et al., "An efficient biometric-based algorithm using heart rate variability for securing body sensor networks," Sensors, vol. 15, no. 7, pp. 1506715089, 2015.

[3] P. Chahuara, A. Fleury, F. Portet, and M. Vacher, "On-line human activity recognition from audio and home automation sensors: comparison of sequential and non-sequential models in realistic Smart Homes," Journal of Ambient Intelligence and Smart Environments, vol. 8, no. 4, pp. 399-422, 2016.

[4] R. Pan, D. Chua, J. S. Pathmasuntharam, and Y. P. Xu, "An opportunistic relay protocol with dynamic scheduling in wireless body area sensor network," IEEE Sensors Journal, vol. 15, no. 7, pp. 3743-3750, 2015.

[5] G. Valenza, L. Citi, C. Gentili, A. Lanatá, E. P. Scilingo, and R. Barbieri, "Characterization of depressive states in bipolar patients using wearable textile technology and instantaneous heart rate variability assessment," IEEE Journal of Biomedical and Health Informatics, vol. 19, no. 1, pp. 263-274, 2015.

[6] Q. Ni, A. B. G. Hernando, and I. P. de la Cruz, "The elderly's independent living in smart homes: a characterization of activities and sensing infrastructure survey to facilitate services development," Sensors, vol. 15, no. 5, pp. 11312-11362, 2015.

[7] B. Taji, S. Shirmohammadi, V. Groza, and I. Batkin, "Impact of skin-electrode interface on electrocardiogram measurements using conductive textile electrodes," IEEE Transactions on Instrumentation and Measurement, vol. 63, no. 6, pp. 1412-1422, 2014.

[8] B.-S. Lin, A. M. Wong, and K. C. Tseng, "Community-based ecg monitoring system for patients with cardiovascular diseases," Journal of Medical Systems, vol. 40, no. 4, article no. 80, pp. 1-12, 2016.

[9] L. Loreto, T. Andrea, D. Lucia, L. Carla, P. Cristina, and R. Silvio, "Accuracy of EASI 12-lead ECGs in monitoring ST-segment and J-point by nurses in the Coronary Care Units," Journal of Clinical Nursing, vol. 25, no. 9-10, pp. 1282-1291, 2016.

[10] F. Miao, Y. Cheng, Y. He, Q. He, and Y. Li, "A wearable context-aware ECG monitoring system integrated with built-in kinematic sensors of the smartphone," Sensors, vol. 15, no. 5, pp. 11465-11484, 2015.

[11] S. Song, M. Rooijakkers, P. Harpe et al., "A low-voltage chopperstabilized amplifier for fetal ECG monitoring with a 1.41 power efficiency factor," IEEE Transactions on Biomedical Circuits and Systems, vol. 9, no. 2, pp. 237-247, 2015.

[12] A. Karilainen, S. Hansen, and J. Muller, "Dry and capacitive electrodes for long-term ECG-monitoring," in Proceedings of 8th Annual Workshop on Semiconductor Advances, vol. 2005, p. 156.

[13] T. Kannaian, R. Neelaveni, and G. Thilagavathi, "Design and development of embroidered textile electrodes for continuous measurement of electrocardiogram signals," Journal of Industrial Textiles, vol. 42, no. 3, pp. 303-318, 2013.

[14] F. Sun, Z. Zhao, Z. Fang, L. Du, and D. Chen, "Design and implementation of a high integrated noncontact ECG monitoring belt," Journal of Fiber Bioengineering and Informatics, vol. 8, no. 1, pp. 37-46, 2015.

[15] H. Li, X. Chen, L. Cao et al., "Textile-based ECG acquisition system with capacitively coupled electrodes," Transactions of the Institute of Measurement and Control, vol. 39, no. 2, pp. 141-148, 2017.

[16] D. Pani, A. Dessì, J. F. Saenz-Cogollo, G. Barabino, B. Fraboni, and A. Bonfiglio, "Fully textile, PEDOT: PSS based electrodes for wearable ECG monitoring systems," IEEE Transactions on Biomedical Engineering, vol. 63, no. 3, pp. 540-549, 2016.

[17] G. Buchberger, R. A. Barb, J. Schoeftner et al., "Transparent, flexible, thin sensor surfaces for passive light-point localization based on two functional polymers," Sensors and Actuators, A: Physical, vol. 239, pp. 70-78, 2016.

[18] M. Vega-Barbas, I. Pau, J. Ferreira, E. Lebis, and F. Seoane, "Utilizing smart textiles-enabled sensorized toy and playful interactions for assessment of psychomotor development on children," Journal of Sensors, vol. 2015, Article ID 898047, 9 pages, 2015.

[19] G. M. Paul, F. Cao, R. Torah, K. Yang, S. Beeby, and J. Tudor, "A smart textile based facial emg and eog computer interface," IEEE Sensors Journal, vol. 14, no. 2, pp. 393-400, 2014.

[20] G. Mattana, T. Kinkeldei, D. Leuenberger et al., "Woven temperature and humidity sensors on flexible plastic substrates for 
e-textile applications," IEEE Sensors Journal, vol. 13, no. 10, pp. 3901-3909, 2013.

[21] G. Cho, K. Jeong, M. J. Paik, Y. Kwun, and M. Sung, "Performance evaluation of textile-based electrodes and motion sensors for smart clothing," IEEE Sensors Journal, vol. 11, no. 12, pp. 3183-3193, 2011.

[22] J. Zięba, M. Frydrysiak, L. Tesiorowski, and M. Tokarska, “Textronic clothing to ECG measurement," in 2011 IEEE International Symposium on Medical Measurements and Applications, MeMeA 2011, Italy, May 2011.

[23] X. Xiao, T. Hua, L. Li, and J. Wang, "Geometrical modeling of honeycomb woven fabric architecture," Textile Research Journal, vol. 85, no. 16, pp. 1651-1665, 2015.

[24] X. Xiao, T. Hua, J. Wang, L. Li, and W. Au, "Transfer and mechanical behavior of three-dimensional honeycomb fabric," Textile Research Journal, vol. 85, no. 12, pp. 1281-1292, 2015.

[25] W. Wu, H. Zhang, S. Pirbhulal, S. C. Mukhopadhyay, and Y.T. Zhang, "Assessment of biofeedback training for emotion management through wearable textile physiological monitoring system," IEEE Sensors Journal, vol. 15, no. 12, pp. 7087-7095, 2015.

[26] A. K. Belchandan, K. Deshmukh, and J. Kumar, "Removal of noises in ECG signal by using digital FIR-IIR filter in VHDL," Digital Signal Processing, vol. 8, no. 5, pp. 135-139, 2016.

[27] A. Gupta and S. Bhandari, "ECG noise reduction by different filters: a comparative analysis," International Journal of Research in Computer and Communication Technology Advance Technology, vol. 4, no. 7, pp. 424-431, 2015.

[28] A. Sahu and J. Kumar, "Filtering of ECG signal using adaptive and non adaptive filters," Manager's Journal on Digital Signal Processing, vol. 4, no. 1, pp. 1-2, 2016.

[29] K. K. Patro and P. R. Kumar, "De-noising of ECG raw signal by cascaded window based digital filters configuration," in IEEE Power, Communication and Information Technology Conference, PCITC 2015, pp. 120-124, India, October 2015. 


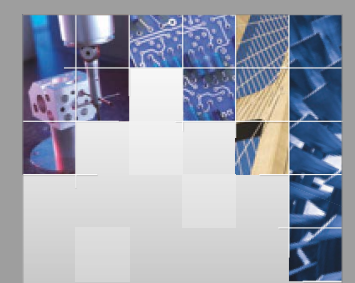

\section{Enfincering}
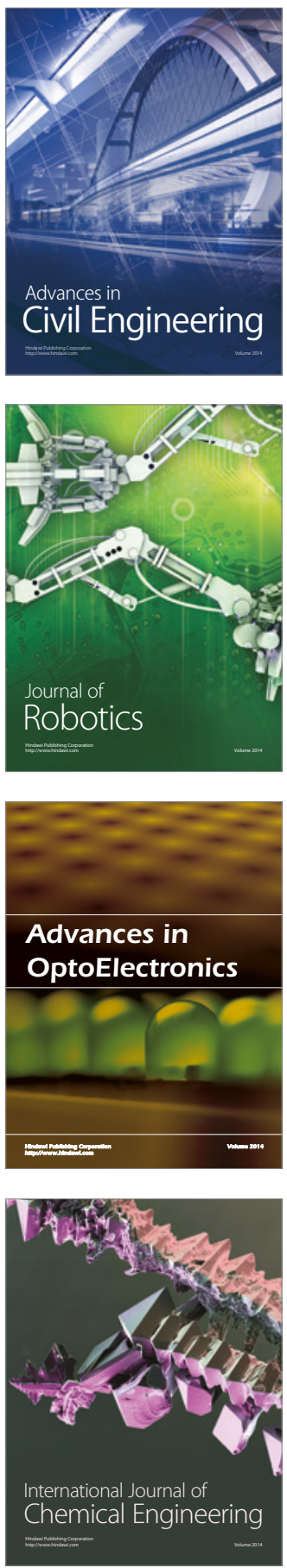

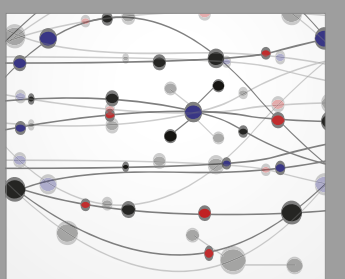

The Scientific World Journal

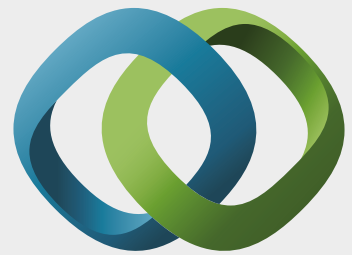

\section{Hindawi}

Submit your manuscripts at

https://www.hindawi.com
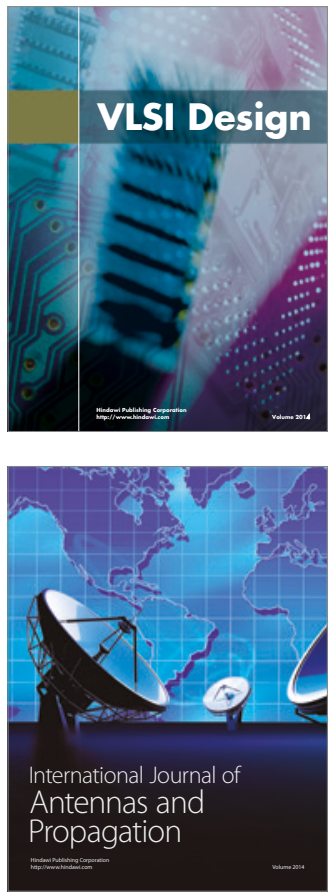

\section{Rotating}

Machinery
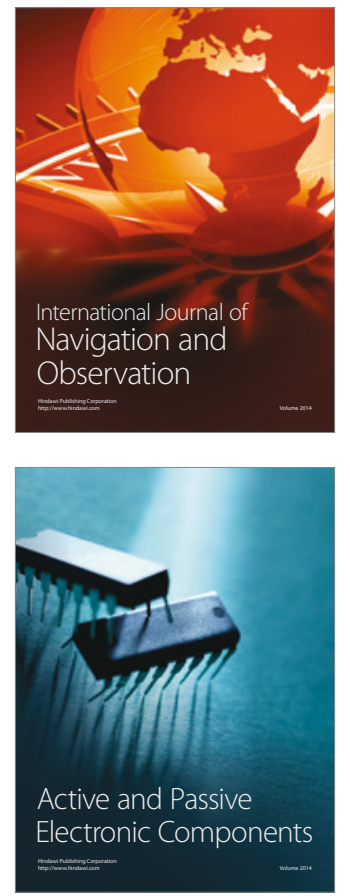
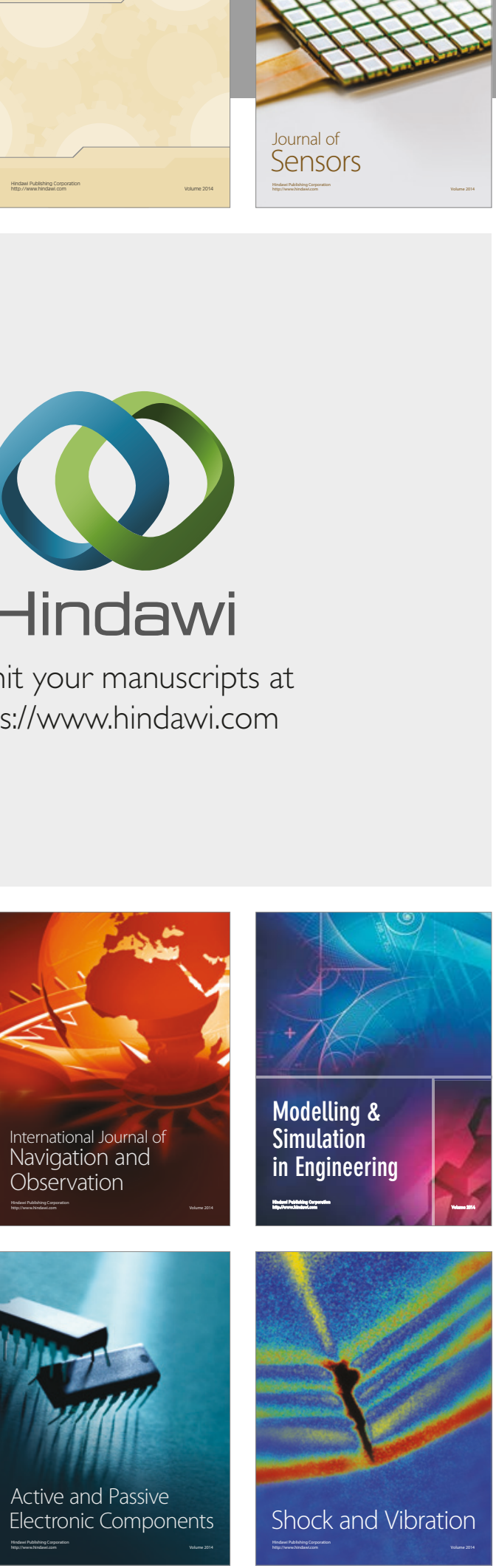
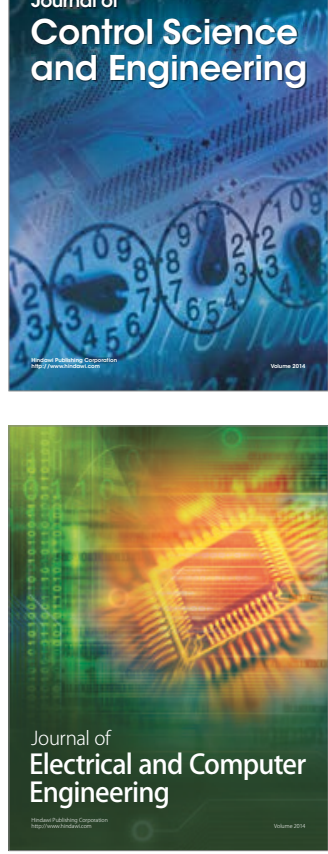

Distributed

Journal of

Control Science

and Engineering
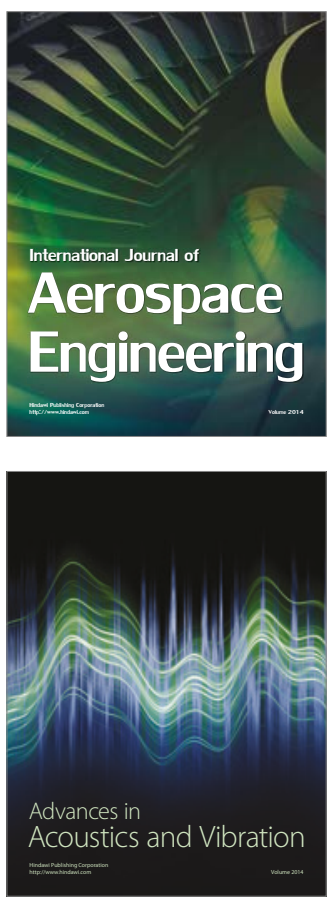

Sensor Networks 